\title{
Chronic Contained Rupture of the Abdominal Aortic Aneurysm
}

\author{
Nabil A Al-zoubi (D) \\ Mahmoud R Mahafdah (D) \\ Omar Albawaih (D)
}

Department of General Surgery, Vascular Surgery, Jordan University of Science and Technology, Irbid, Jordan
Correspondence: Nabil A Al-zoubi Tel +962 07955774637

Email dr_nabeil_z3bi@yahoo.com; nazoubi@just.edu.jo
Background: The most lethal complication of the abdominal aortic aneurysm (AAA) is rupture (rAAA). A triad of abdominal or back pain, a pulsating mass in the abdomen, and decrease in blood pressure is mostly diagnostic. However, this presentation may not be complete due to either an impalpable aneurysm or atypical symptoms which leads to difficulties in diagnosis and delayed management. Chronic contained rupture of AAA (CCRAAA) is a rare but well-recognized condition. Its diagnosis may be difficult because of the atypical and chronic nature of the symptoms. The aim of this study is to investigate the incidence and to highlight the importance of this less common presentation of rAAA.

Methods: Patients who presented to King Abdullah University Hospital (KAUH) with infrarenal AAAs (elective and emergency) from January 2014 to April 2021 were prospectively collected. Patients with CCR-AAA were identified and evaluated in terms of demographic data, associated comorbidities, presentation, treatments, and outcomes.

Results: A total number of 85 patients were admitted with an infra-renal AAA. Seventeen patients $(20.0 \%)$ had rAAA, of them only 5 patients $(29.4 \%)$ were diagnosed with CCRAAA. CCR-AAAs represent $5.9 \%$ of cases with AAA. CCR-AAAs were all in men, with a mean age \pm SD of $73.1 \pm 8.3$. Two patients $(40.0 \%)$ had abdominal pain, 2 patients $(40.0 \%)$ had back pain and one patient $(10.0 \%)$ had no symptoms at the time of diagnosis.

Conclusion: CCR-AAA represents $5.9 \%$ of all AAA and $29.4 \%$ of rAAA. To prevent this potentially fatal condition, awareness is required especially in old males with abdominal or back pain.

Keywords: AAA, rupture, abdominal pain, back pain

\section{Introduction}

AAA is more than $50 \%$ dilatation in the diameter of the abdominal aorta. ${ }^{1}$ It is usually asymptomatic, symptomatic non-ruptured, and ruptured. ${ }^{2}$ Its rupture is commonly fatal with an overall mortality rate of nearly $90 \%{ }^{1,3,4}$ Rarely, rAAA may be effectively "sealed off" by the surrounding peritoneum, which leads to a "chronically" contained retroperitoneal hematoma which resulted in CCR-AAA. ${ }^{5}$ This condition was first described by Szilagyi in 1961 as reported by Ando $\mathrm{M}$ et al. ${ }^{6}$ However, Jones introduced the term "CCR-AAA" as described by Davidovic LB et al. ${ }^{5}$ Then, it became a well-documented subtype of AAA rupture. ${ }^{5,7}$ The aim of this article is to investigate the incidence of CCRAAA and to highlight the importance of this less common presentation of rAAA.

\section{Methods}

Institutional review board of Jordan University of Science and Technology (JUST) approved this study. Due to the lack of a breach of confidentiality, patient informed 
consents were waived. This research was carried out in line with the Helsinki Declaration. Patients who presented to KAUH with infra-renal AAA (elective and emergency) from January 2014 to April 2021 were prospectively identified and reviewed. The diagnosis of both AAAs and rAAAs was mainly confirmed by multi-detector computed tomography angiography (CTA). Criteria used to diagnose CCR-AAAs were the presence of AAA, history of abdominal pain, absence of low blood pressure with no drop in hemoglobin level, and CT scans with or without contrast that demonstrate organized retroperitoneal hematoma. ${ }^{8}$ Medical records were reviewed in terms of demographic data, associated comorbidities, presentation, treatments, and outcomes.

\section{Results}

Eighty-five patients with an infra-renal AAA were identified. Seventeen patients $(20.0 \%)$ had rAAA, of them only 5 patients (29.4\%) diagnosed with CCR-AAA. CCRAAAs represent $(5.9 \%)$ of cases with AAA. CCR-AAAs were all in men, with a mean age $\pm \mathrm{SD}$ of $73.1 \pm 8.3$. Four patients $(80.0 \%)$ had hypertension, 2 patients $(40.0 \%)$ were diabetic and all were smokers. Two patients (40.0\%) had abdominal pain, 2 patients (40.0\%) had back pain and one patient $(10.0 \%)$ had no symptoms at the time of diagnosis who was investigated for renal symptoms. All of them were clinically stable with no acute distress or change in hemoglobin level on admission. Two patients refused any type of surgery or interventions. Two patients underwent endovascular aortic repair (EVAR) and one patient underwent open aortic repair (OAR) within 24 hours of the diagnosis. Table 1 demonstrates clinical features of patients with CCR-AAAs.

\section{Discussion}

The CCR-AAA is a rare condition which represents $2.7 \%$ to $4 \%$ of all operated infra-renal AAAs. ${ }^{7,9}$ Not like free rAAA which often presents with a pulsatile abdominal mass, abdominal pain, and hypotension, ${ }^{4,10}$ CCR-AAAs may present with different symptoms which are usually indefinite and resulted from compression to the nearby structures rather than changes in the patient's hemodynamics. ${ }^{11}$ Clinical stability of patients for long periods usually resulted in a delayed diagnosis unless awareness of this potentially lethal entity is kept. ${ }^{11}$

Findings on imaging studies for rAAA are usually clear; however, small rAAA can be mistaken for bowel unopacification, lymphadenopathy, or fibrosis of the peri-
Table I Clinical Features of 5 Patients with Chronic Contained Rupture of AAA

\begin{tabular}{|c|c|}
\hline Variables & $\mathbf{n}=\mathbf{5}$ \\
\hline Mean age in years \pm SD & $73.1 \pm 8.3$ \\
\hline Males (\%) & $5(100)$ \\
\hline Median Length of history/days & 35 \\
\hline $\begin{array}{l}\text { Duration from presentation to diagnosis, Hours } \\
\text { Mean } \pm \text { SD }\end{array}$ & $7.1(2.1)$ \\
\hline \multicolumn{2}{|l|}{ Cardio-vascular history (\%) } \\
\hline Hypertension & $4(80)$ \\
\hline Myocardial infarction & I (20) \\
\hline Cerebral vascular disease & $0(0)$ \\
\hline Peripheral vascular disease & $2(40)$ \\
\hline Smoking & $5(100)$ \\
\hline \multicolumn{2}{|l|}{ Presenting symptoms and signs (\%) } \\
\hline Abdominal pain & $2(40)$ \\
\hline Back pain & $2(40)$ \\
\hline Pulsating Abdominal mass & I (20) \\
\hline Asymptomatic & I (20) \\
\hline \multicolumn{2}{|l|}{ Site of rupture $n(\%)$} \\
\hline Anterior & $2(40)$ \\
\hline Posterior & $3(60)$ \\
\hline \multicolumn{2}{|l|}{ Treatment (\%) } \\
\hline EVAR & $2(40)$ \\
\hline OAR & I (20) \\
\hline Refused & $2(40)$ \\
\hline Mortality (\%)* & $0(0)$ \\
\hline Morbidity & $0(0)$ \\
\hline \multicolumn{2}{|l|}{ EVAR-related } \\
\hline \multicolumn{2}{|l|}{ OAR-Related } \\
\hline Previously known AAA (\%) & $0(0)$ \\
\hline
\end{tabular}

Note: *Only for the three patients who underwent EVAR and OAR.

Abbreviations: SD, standard deviations; AAA, abdominal aortic aneurysm; OAR, open aortic repair; EVAR, endo-vascular aortic repair.

aneurysmal tissue; therefore, vigilant inspection of the AAA morphology is important in detecting CCR-AAA. ${ }^{7}$ CTA is an important and the most reliable diagnostic tool for the assessment of the AAAs, the presence of complications, and the correlations between the aneurysmal sac and the major vessels as well as the nearby abdominal structures. ${ }^{12,13}$ Features of CCR-AAA on CTA that led to the correct diagnosis include disruption of the true aneurysmal wall calcification rim, organized soft tissue density near to the AAA, obscured psoas muscle, shifted abdominal structures, and lack of contrast material in the retroperitoneal hematoma in some images. ${ }^{6,11}$ 


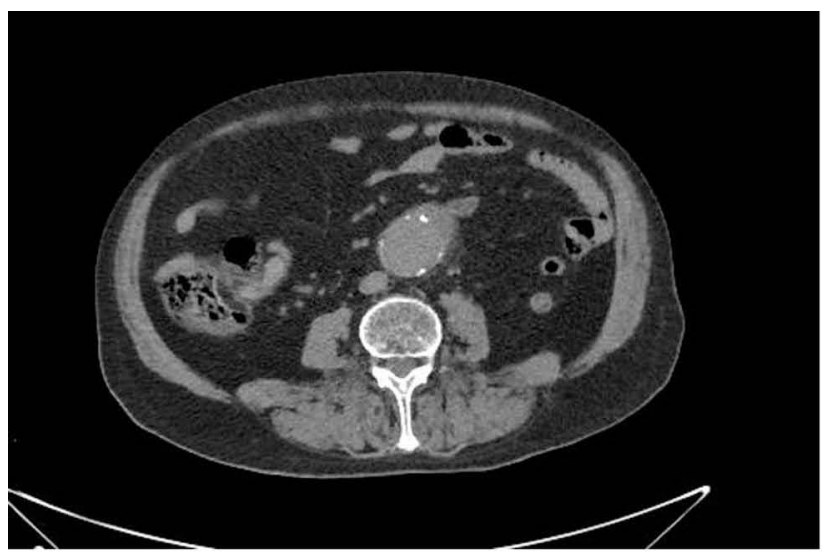

A

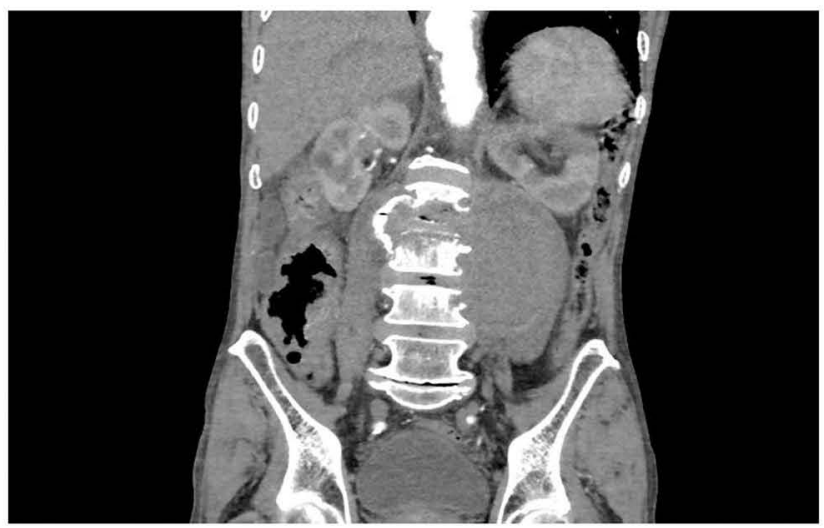

C

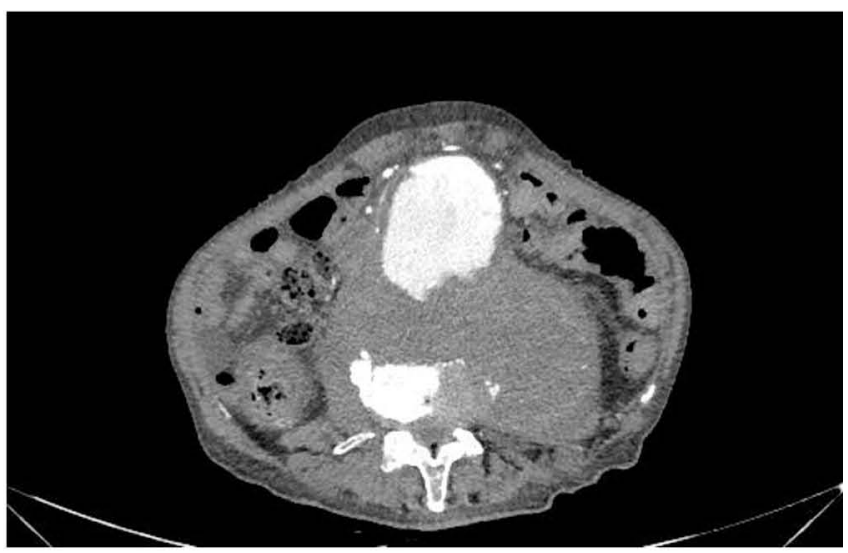

B

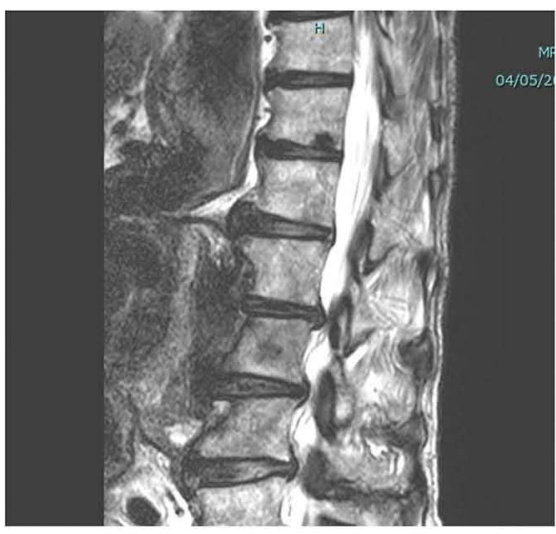

D

Figure I (A) CT without contrast showing anterior rAAA with hematoma outside the rim of calcification. (B) Posterior rAAA with huge hematoma. (C) CCR-AAA with vertebral destruction. (D) MRI showing CCR-AAA with vertebral destruction.

Proper treatment depends on distinction between CCRAAA and free rAAA. $^{8}$ CCR-AAAs have a high risk to progress to free rupture which carry a high morbidity and mortality, therefore an urgent management and intervention are essential. ${ }^{9}$ However, if diagnosed and managed properly, they have comparable morbidity and mortality to that associated elective interventions. Treatment options include either OAR or EVAR. ${ }^{9}$

In this study, we identified 5 patients with CCR-AAAs. All of them were old males with no previous history of AAA. Two patients were presented with abdominal pain of more than 4 weeks, two patients were presented with back pain of more than 2 months, and one patient was asymptomatic at the time of diagnosis. Four patients reported an isolated episode of abdominal pain before the chronic nature of their symptoms. All of them were stable vital signs and showed no significant decrease in hemoglobin level on admission and during follow-up. Physical examination revealed a pulsatile abdominal mass in only one patient
(20\%). Four patients were evaluated with CTA and one patient had impaired kidney functions and evaluated by CT scan without contrast. Imaging studies revealed infra-renal AAA with features of CCR-AAA (Figure 1A-D). Two patients were refused treatment and lost follow-up. The remaining 3 patients were operated on within 24 hours of evaluation. Two patients underwent urgent EVAR (one patient with CO2-EVAR). In both cases, EVAR was done under general anesthesia. The open bilateral common femoral arteries approach was used to place the covered stents into the infra-renal aorta and both common iliac arteries. Both procedures were successful with no postoperative complications. One patient underwent open aortic repair OAR due to unsuitable anatomy for EVAR. The aneurysm was isolated, clamped and opened (Figure 2). A posterior wall semi-circular defect (width $3 \mathrm{~cm} \times$ length $3.5 \mathrm{~cm}$ ) was found in the aneurysmal sac $3 \mathrm{~cm}$ below the renal arteries which contained a thrombosed old organized hematoma (Figure 3). An infra-renal aorto-bi-external iliac 


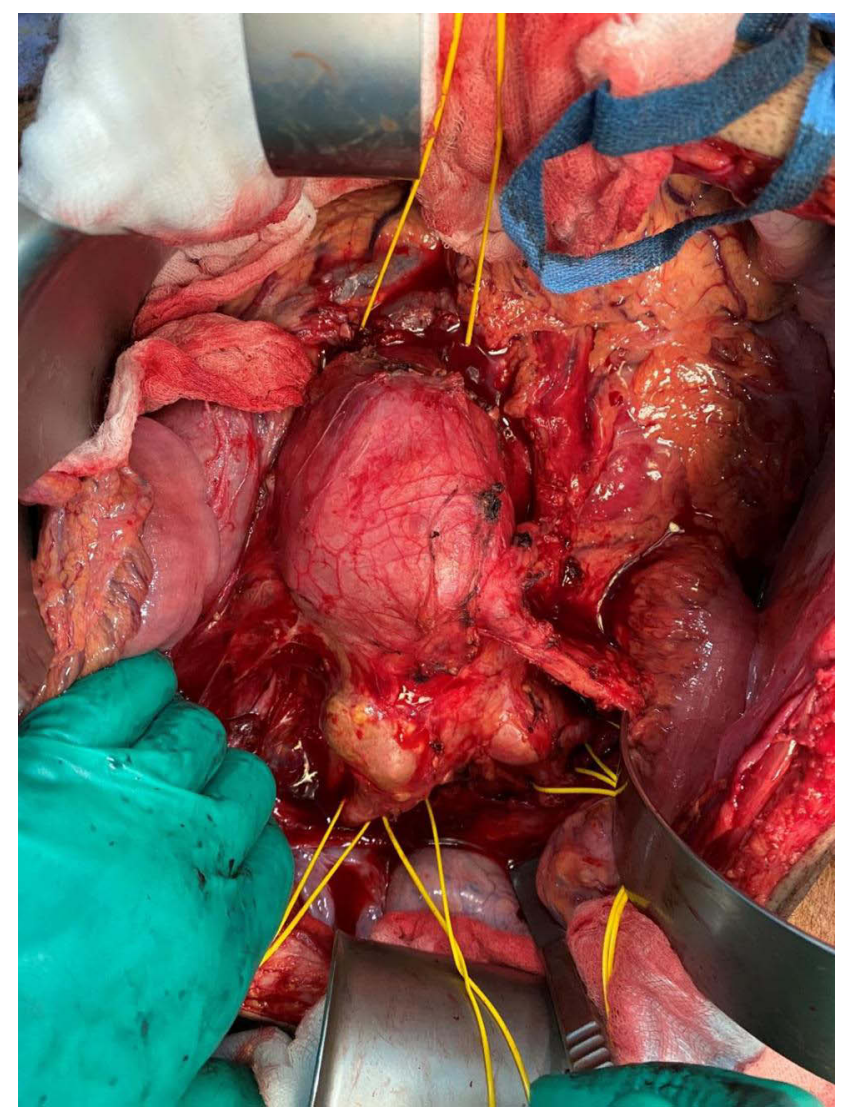

Figure 2 Intra-operative infra-renal aorto-bi-iliac AAA.

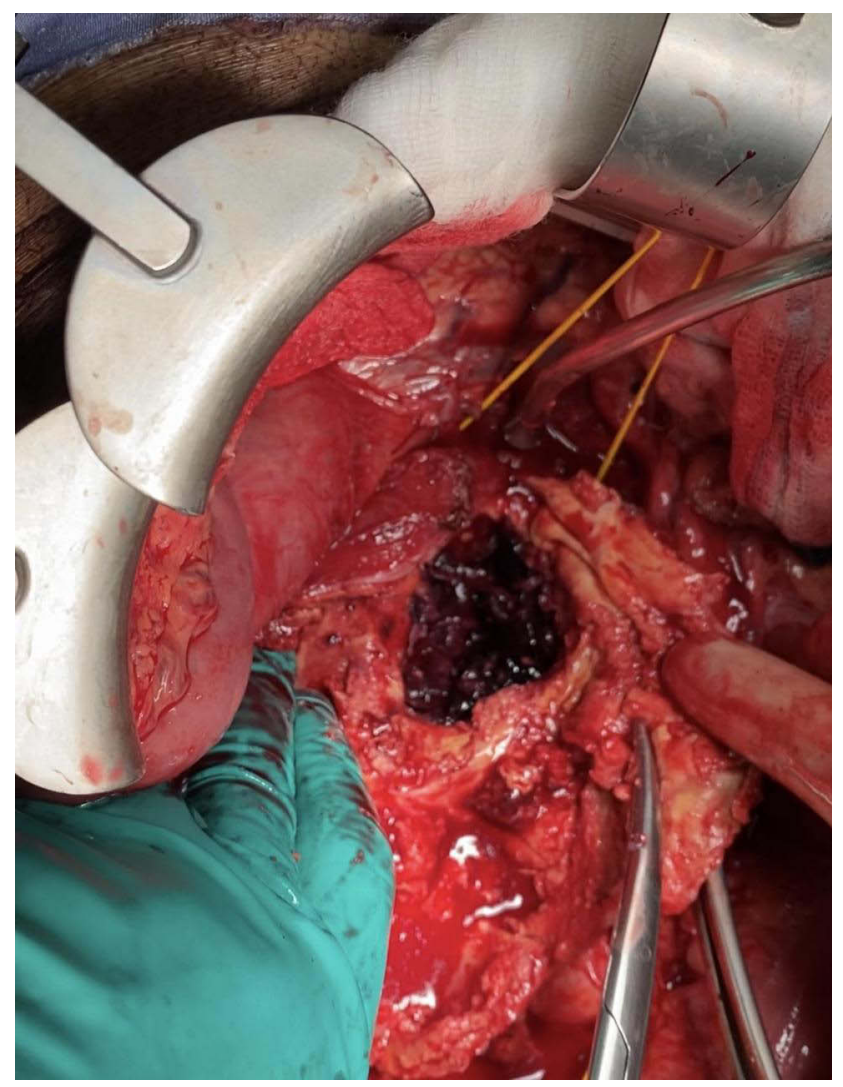

Figure 3 Aneurysmal sac with posterior wall rupture and chronic hematoma.

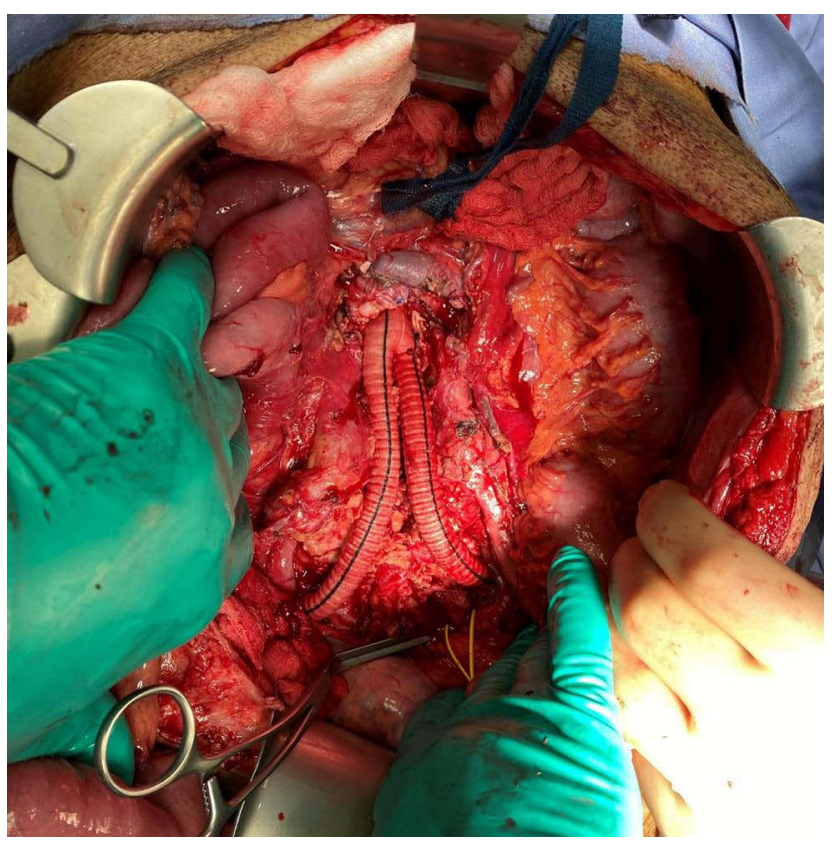

Figure 4 Aorto-bi-iliac dacron-graft bypass.

Dacron-graft bypass with right internal iliac artery reimplantation was done (Figure 4) with uneventful recovery.

The limitation of the study was the small number of patients with CCR-AAA which leads to the inability to compare patient's characteristics and risk factors between these patients and patients with acute rAAA.

\section{Conclusions}

CCR-AAA represents $5.9 \%$ of all AAA and $29.4 \%$ of rAAA. To prevent this potentially fatal condition, awareness is required especially in old males with abdominal or back pain.

\section{Disclosure}

The authors report no conflicts of interest in this article.

\section{References}

1. Riambau V, Guerrero F, Montañá X, Gilabert R. Abdominal aortic aneurysm and renovascular disease. Rev Esp Cardiol. 2007;60 (6):639-654. doi:10.1157/13107121

2. Booth MI, Galland RB. Chronic contained rupture of an abdominal aortic aneurysm: a case report and review of the literature. EJVES Extra. 2002;3:33-35. doi:10.1053/ejvx.2002.0128

3. Wadgaonkar AD, Black III JH, Weihe EK, Zimmerman SL, Fishman EK, Johnson PT. Abdominal aortic aneurysms revisited: MDCT with multi-planar reconstructions for identifying indicators of instability in the pre and postoperative patient. RadioGraphics. 2015;35:254-268. doi:10.1148/rg.351130137

4. Banerjee A. Atypical manifestations of ruptured abdominal aortic aneurysms. Postgrad Med J. 1993;69:6-11. doi:10.1136/ pgmj.69.807.6 
5. Davidović LB, Lotina SI, Cinara IS, Zdravković D, Simić TA, Djorić P. Chronic rupture of abdominal aortic aneurysms. Srp Arh Celok Lek. 1998;126(5-6):177-182.

6. Ando M, Igari T, Yokoyama H, Satokawa H. CT features of chronic contained rupture of an abdominal aortic aneurysm. Ann Thorac Cardiovasc Surg. 2003;9(4):274-278.

7. Alshafei A, Kamal D. Chronic contained abdominal aortic aneurysm rupture mimicking vertebral spondylodiscitis: a case report. Ann Vasc Dis. 2015;8(2):113-115. doi:10.3400/avd.cr.15-00010

8. Schwartz SA, Taljanovic MS, Smyth S, O'Brien MJ, Rogers LF. CT findings of rupture, impending rupture, and contained rupture of abdominal aortic aneurysms. AJR. 2007;188:57-62. doi:10.2214/ AJR.05.1554

9. Lai CC, Tan CK, Chu TW, Ding LW. Chronic contained rupture of an abdominal aortic aneurysm with vertebral erosion. CMAJ. 2008;8(178):995-996. doi:10.1503/cmaj.070332
10. Higgins R, Peitzman AB, Reidy M, Stapczynski S, Steed DL, Webster MW. Chronic contained rupture of an abdominal aortic aneurysm presenting as a lower extremity neuropathy. Ann Emerg Med. 1988;17(3):284-287. doi:10.1016/S0196-0644(88)80125-2

11. Singla V, Virmani V, Modi M, Kalra N, Khandelwal N. Chronic rupture of abdominal aortic aneurysm with vertebral erosion: an uncommon but important cause of back pain. Ann Vasc Surg. 2014;28:1931-1934. doi:10.1016/j.avsg.2014.07.006

12. Yuksekkaya R, Koner AE, Celikyay F, Beyhan M, Almus F, Acu B. Multidetector computed tomography angiography findings of chronic-contained thoracoabdominal aortic aneurysm rupture with severe thoracal vertebral body erosion. Case Rep Radiol. 2013;2013:596517. doi:10.1155/2013/596517

13. Ozkacmaz S. Computed tomography and magnetic resonance imaging findings of chronic contained rupture of an abdominal aortic aneurysm leading to vertebral destruction and total thrombosis: a case report. J Vasc Med Surg. 2017;5:5. doi:10.4172/2329-6925.1000339

\section{Publish your work in this journal}

The Open Access Emergency Medicine is an international, peerreviewed, open access journal publishing original research, reports, editorials, reviews and commentaries on all aspects of emergency medicine. The manuscript management system is completely online and includes a very quick and fair peer-review system, which is all easy to use. Visit http://www.dovepress.com/testimonials.php to read real quotes from published authors. 